\title{
The System for Early Detection of Heart-Attack
}

\author{
Swati Chandurkar \\ Computer Department \\ Pimpri Chinchwad College of \\ Engineering \\ Pune
}

\author{
Shraddha Arote \\ Computer Department \\ Pimpri Chinchwad College of \\ Engineering \\ Pune \\ Vaishnavi Kakade \\ Computer Department \\ Pimpri Chinchwad College of Engineering \\ Pune
}

\author{
Snehal Chaudhari \\ Computer Department \\ Pimpri Chinchwad College of \\ Engineering \\ Pune
}

\begin{abstract}
In the recent years, heart attack has become an alarming disease. Heart diseases have become one of the leading causes of death. In India, the number of deaths caused by heart attacks is about $25 \%$ of the total death. This happens due to the delay in detecting the symptoms or lack of early diagnosis. This can be avoided by integrating mobile computing technologies with health care systems, which will lead to detect abnormal heart rates and predict heart attacks before it occurs. Heart disease is a major cause of morbidity in the modern society. The earlier system detect the risk of heart attack using only limited parameter which are ECG, pulserate. Hence it cannot guarantee the risk for other symptoms like left shoulder pain, chest pain and etc. So the proposed system did consider all the parameter which can be a symptom of heart attack and hence provide a accurate risk detection system. The proposed system describes a heart attack self-test mobile application that allows potential victims, without the intervention of any medical specialist, to quickly assess whether they are having a heart attack.
\end{abstract}

\section{General Terms}

Naïve Bayes Classifier, Framingham Risk Score

\section{Keywords}

Pulse rate sensor, ECG, smartphone, Blood pressure sensor, Real-time monitoring.

\section{INTRODUCTION}

In the recent years, heart attack has become an alarming disease. Heart diseases have become one of the leading causes of death. In India, the number of deaths caused by heart attacks is about $25 \%$ of the total death. This happens due to the delay in detecting the symptoms or lack of early diagnosis. This can be avoided by integrating mobile computing technologies with health care systems. This will lead to detect the abnormal heart rhythms and predict heart attacks before it occurs. Heart disease is a major cause of morbidity in the modern society. A heart attack caused when one or more of your coronary arteries are blocked. As time passes, a coronary artery can narrow from the build-up of various substances, including cholesterol. This condition is called as coronary artery disease, and the reason behind heart attack.

The heart muscle requires a constant oxygen-rich blood supply to nourish it. The coronary arteries provide the heart with oxygen-rich blood supply. Fatty matter, cholesterol, calcium, proteins, and inflammatory cells build up inside the arteries to form plaques of different sizes. The plaque deposits on the outside and soft and mushy on the inside.

When the plaque is rigid, the outer shell cracks (plaque rupture), platelets (disc-shaped molecules in the blood that aid clotting) come to the area and blood clots form all over the plaque. If a blood clot completely blocks the artery then the heart muscle becomes "starved" for oxygen. Within a short time, death of heart muscle cells occurs which causes permanent damage. This is a heart attack.

A feeling of nausea, tightness, pain, squeezing, or aching in the chest or arms which spreads to the neck, jaw, or back can be a sign that a person is having a heart attack.

The possible signs and symptoms of a heart attack are as follows:

- coughing

- nausea

- vomiting

- crushing chest pain

- dizziness

- $\quad$ shortness of breath called dyspnea

- face seeming gray in colour

- a feeling of terror that life is ending

- feeling awful, generally

- restlessness

- feeling clammy and sweaty

- $\quad$ shortness of breath

The earlier system detect the risk of heart attack using only limited parameter which are ECG, pulse-rate. Hence it cannot guarantee the risk for other symptoms like left shoulder pain, chest pain and many from mentioned above. Proposed system considers all the above mentioned symptoms and predicts the risk of heart attack earlier. First stage of system will continuously monitor patient's heart-beats, ECG and if any risk is identified, continue to the stage II. In second stage, the system will ask set of questions, which will be criteria to identify the risk of having heart attack. Hence considering all the possible symptoms of heart attack, system will predict the risk of heart attack.

\section{RELATED WORK}

There are existing technologies which include the heart attack detection system using android and a self-test to detect a heart attack using phone and sensors. But they mainly focus on personal ECG monitoring. [1] The research describes the how 
to develop a wireless sensor network that can continuously monitor and detect heart attack disease experienced in patients at remote areas. A wearable wireless sensor system is designed to gather and transmit the signals to the patient's device continuously. [2]In this paper, Gowrishankar S., PhD Prachita M. Y. Arvind Prakash had proposed a remote sensing parameter of the human body which consists of pulse and temperature. The parameters that are used for sensing as well as for monitoring will send the data through wireless sensors by a app based observing helps to keep track of the regular health status of a patient. The sensed data will be continuously gathered in a database and will be used to inform patient to any unnoticed problems to undergo possible diagnosis. A simple approach is proposed in [3] this paper is to predict risk of developing heart attack using smart phone and data mining. A user friendly Android application was developed with the help of clinical data which is obtained from patients who admitted with chest pain in a cardiac health care. Upon user input of risk factors, the application categorizes the level of risk of heart attack of the user as high, low or medium. Hence the risk of heart attack is predicted and categorized into three categories as high, low and medium. The research paper [4] describes the use of a device which can perform the dual function to detect a heart attack without the intervention of any specialist for victim and also report the emergency services. The device through sensors continuously monitor the patient's heart beat and notify the emergency contacts if any kind of risk is found.

\section{PROPOSED SYSTEM}

For heart monitoring systems, the method usually consists of a sensor which is carried by the patient that is connected to a backbone network via a gateway node. The patient and doctors both can monitor and diagnose heart rate information by a graphical user interface (GUI). In the case of an emergency situation, an alert message is sent to a laptop or a personal computer in the closest hospital or to the emergency contact filled by the user.

The proposed application consists of two main components: i) the sensor and ii) questionnaire.

\section{Sensors:}

To detect the heart rate the commercially available sensor is, the Pulse Sensor AMPED. It is a simple optical heart rate sensor with amplification and noise cancellation circuitry making it fast and easy. It is very specific sensor because of its reliability, high usability and good performance. Also, it is of a small size and a modest price. An important issue to consider while implementing sensors is power consumption.

The Blood Pressure Sensor (Vernier) is a non-invasive sensor designed to measure human blood pressure. It measures systolic, diastolic and mean arterial pressure using the oscillometric technique. Pulse rate is also reported.
The electrocardiogram (ECG) is a tool that is routinely used for assessing the electrical and muscular functions of the heart. The AD8232 sensor module is an integrated signal conditioning block for ECG and other biopotential measurement applications. It is mainly designed to extract, amplify, and filter small biopotential signals in the presence of noisy conditions those created by motion or remote electrode placement.

2. Questionnaire: The questionnaire consists of set of questions which we the symptoms of heart attack. The most commonly reported prodromal symptoms in male and female heart patients are:

- unusual fatigue: $100 \%$

- anxiety: $100 \%$

- arm pain/discomfort: $86 \%$

- shortness of breath: $86 \%$

- nausea, vomiting, indigestion: $71 \%$

- chest pain/discomfort: $57 \%$

- jaw pain: $43 \%$

- back/shoulder blade pain: $43 \%$

- dizziness: $43 \%$

- headache: $43 \%$

- $\quad$ sleep disturbance: $29 \%$

According to the prodromal symptoms mentioned above, the questionnaire is consist of the questions based on these symptoms only. For example, do you feel severe jaw pain?

The risk for these two components is calculated based on this two algorithms:

\section{NAÏVE BAYES CLASSIFIER}

Naive Bayes is a simple technique for constructing classifiers models which assign class labels to problem instances which can be represented as vectors of feature values, where the class labels are drawn from some finite set. Naive Bayes is one of the data mining technique which shows better result and accuracy. None of the system predicts risk of heart attack based on risk factors such as age, diabetes, family history, hypertension, high cholesterol, tobacco smoking, alcohol intake, obesity or physical inactivity, etc. System based on these risk factors would not only help to reduce the death rate of Heart attack patient in the rural areas but it would also give patients a notification(warning) and suggestion about the probable presence of heart attack even before patient visits a hospital or goes for costly medical checkups.

Naive Bayes is a conditional probability model in which given a problem instance to be classified and represented by a 


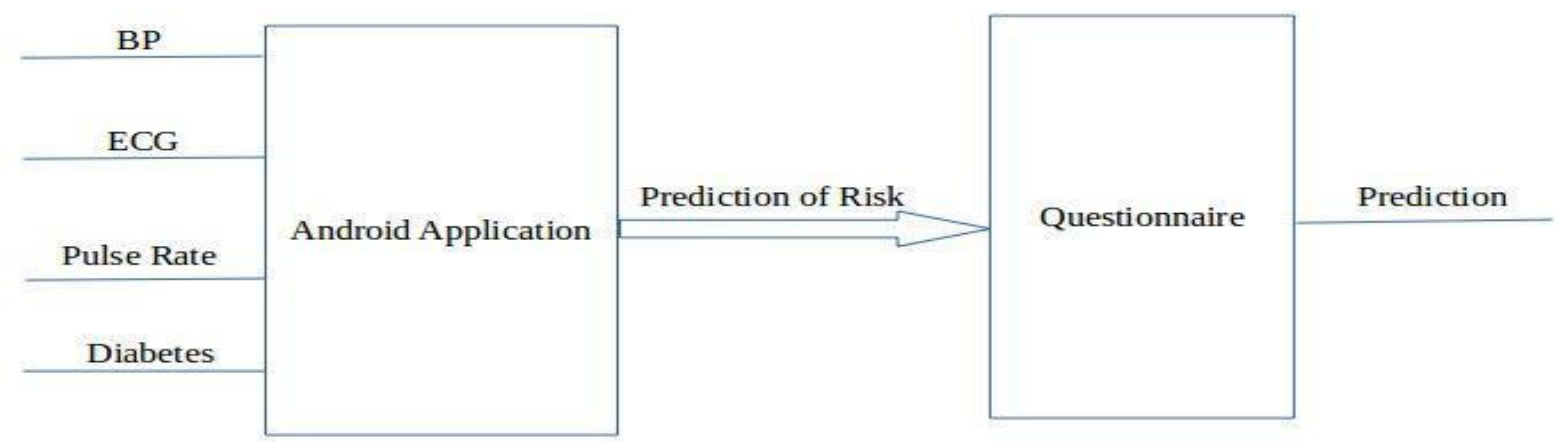

\section{Fig 1: System Architecture}

vector $\mathrm{x}=\{\mathrm{x} 1, \mathrm{x} 2, \mathrm{x} 3 \ldots \ldots \mathrm{xn}\}$ representing some $n$ features (independent variables), it assigns to this instance probabilities $\mathrm{p}(\mathrm{Ck} \mid \mathrm{x} 1, \mathrm{x} 2 \ldots \ldots \mathrm{xn})$

USING BAYES CLASSIFIER THE CONDITIONAL PROBABILITY CAN BE DECOMPOSED AS

$\mathrm{P}(\mathrm{CK} \mid \mathrm{X})=\mathrm{P}(\mathrm{CK}) \cdot \mathrm{P}(\mathrm{X} \mid \mathrm{CK}) / \mathrm{P}(\mathrm{X})$

\section{FRAMINGHAM RISK SCORE:}

The Framingham Risk Score is one of the number scoring systems which is used to determine an individual's chances of developing heart attack. Heart attack risk scoring systems give an estimate of the probability that a person will develop heart attack within a specified amount of time. Because they give an indication of the risk of developing heart attack, they also indicate who is most likely to benefit from prevention.

Hence the system can be explained as

1. ECG, Pulse rate sensor will detect real time data of patients continuously. In this risk can be categorized as low, medium, high.

2. If risk is high it will immediately notify patients and doctors also.

3. If risk is low or medium system will ask questions to patients about minor symptoms like jaw pain, arm pain, headache etc.

4. By using this questions system will again recalculate risk of heart attack.

5. At the end, System will show actual risk of heart attack. Again if risk is high it will immediately notify patients and doctors also.

The diagrammatic representation is fig

\section{CONCLUSION}

In India the number of deaths caused by heart attacks is about $25 \%$ of the total death. This occurs due to the delay in detecting the symptoms or lack of early diagnosis of heart related problems. This can be avoided by integrating mobile computing technologies with health care systems. Thus we are designing and developing real-time processing heart rate counting system which is able to notice any heart anomalies and heart attack by tracking heart rate of a user at early stage.

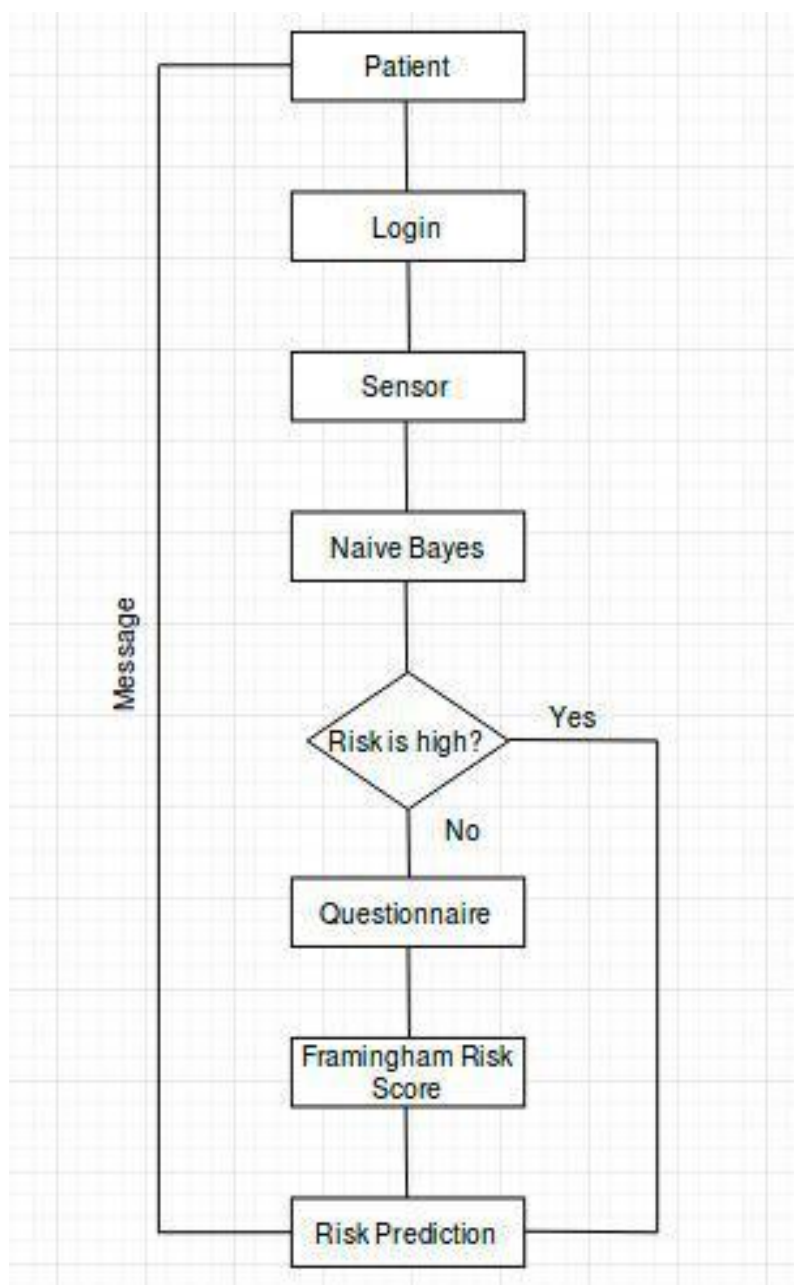

Fig 2 : Data Flow Diagram

\section{FUTURE WORK}

The system is a real time monitoring system which will monitor the pulse-rate, ECG, BP of patient continuously. Also proposed system consider all the parameters like left shoulder pain ,chest pain and etc. which can be a symptom of heart attack and hence provide a accurate risk detection system. For more accuracy and faster risk prediction, system can capture photo or short video of user facing on camera. Due to this, system captures the picture of user and immediately alert will be given. 


\section{REFERENCES}

[1] Raghvendra Tiwari, Kajol Kumari Vishwakarma, Poonam Singh Surbhi, Raj Kumar, Anamika Gupta, "Wireless Heart Attack Detection System", February 2017.

[2] Gowrishankar S., PhD Prachita M. Y. Arvind Prakash, "IoT based Heart Attack Detection, Heart Rate and Temperature Monitor", July 2017.

[3] M. Raihan, Saikat Mondal, Arun More, Pritam Khan Boni, Md. Omar Faruqe Sagor, Smartphone Based Heart Attack Risk Prediction System with Statistical Analysis and Data Mining Approaches, Advances in Science, Technology and Engineering Systems Journal Vol. 2, No. 3, 1815-1822 (2017)

[4] Ashwini Babasaheb Patil, P. A. More, "Heart Disease Detection using Android Application and Internet of Things (IoT)", 2016.

[5] Chao Lia, Xiangpei Hua, Lili Zhangb, "The IoT-based heart disease monitoring system for pervasive healthcare service", 6-8 September 2017.

[6] Shivam Patel, Yogesh Chauhan, "Heart attack detection and Medical attention using Motion Sensing DeviceKinect”, January 2014.

[7] Georg Wolgast, Casimir Ehrenborg, Alexander Israelsson, Jakob Helander, Edvard Johansson and
Hampus Månefjord, ”Wireless Body Area Network for Heart Attack Detection", October 2016.

[8] Ahmed Fawzi Otoom, Emad E. Abdallah, Yousef Kilani, Ahmed Kefaye, "Effective Diagnosis and Monitoring of Heart Disease", 2015.

[9] Mahshid Zomorodi Rad, Saeed Rahati Ghuchani, Kambiz Bahaadinbeigy, Mohammad Mahdi Khalilzadeh,"Real Time Recognition of Heart Attack in a Smart Phone", May 2015.

[10] Peter Leijdekkers and Valérie Gay, "A Self-test to Detect a Heart Attack Using a Mobile Phone and Wearable Sensors", 2015.

[11] Kala John Kappiarukudil, Maneesha Vinodini Ramesh, "Real-Time Monitoring and Detection of "Heart Attack' Using Wireless Sensor Networks", 2010.

[12] Thomas, C. (2018). Can early warning symptoms predict a heart attack?. [online] Heart Sisters. Available at https://myheartsisters.org/2018/03/18/can-early-warningsymptoms-predict-a-heart-attack/ [Accessed 16 Oct. 2018].

[13] Ng, K. (2018). Using AI and science to predict heart failure. [online] IBM Blog Research. Available at: https://www.ibm.com/blogs/research/2017/04/using-aito-predict-heart-failure/ [Accessed 16 Oct. 2018]. 